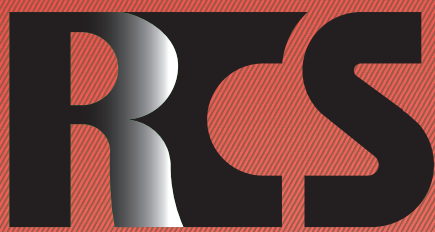

Depósito legal ppi $201502 Z U 4662$

Esta publicación científica en formato digital es continuidad de la revista impresa Depósito Legal: pp $197402 Z$ Z789

- ISSN: 1315-9518 • ISSN-E: 2477-9431

Revista de Ciencias Sociales

Universidad del Zulia. Revista de la Facultad de Ciencias Económicas y Sociales Vol. XXVII,

No. 4,2021

Esta publicación científica en formato digital es continuidad de la revista impresa Depósito Legal: pp $197402 Z 1789$ ISSN: $1315-9518$ 


\title{
Estimulación temprana y desarrollo de habilidades del lenguaje: Neuroeducación en la educación inicial en Ecuador
}

\author{
Coello Villa, Mayra Cecilia*
}

\section{Resumen}

La estimulación temprana es considerada un factor fundamental para desarrollar y potenciar las funciones cerebrales psicomotoras del niño ayudando a fortalecer aspectos cognitivos, lingüísticos, motrices y sociales. El objetivo de la investigación es, analizar los aspectos neurológicos y teóricos que fundamentan el programa de estimulación temprana en niños de educación inicial en Ecuador. El estudio es mixto, empleando técnicas descriptivas e interpretativas con diseño fenomenológico, de campo y correlacional. La población fue de 400 niños de educación inicial I y II de Unidades Educativas de la provincia de BolívarEcuador, utilizando como instrumento la Escala Abreviada de Desarrollo de Ortiz para conocer habilidades y destrezas lingüísticas desde el enfoque de neuroeducación. Los resultados indican que, en la primera evaluación, se mantiene una estimación media, motricidad gruesa (56\%), motricidad fina adaptativa (71\%), audición y lenguaje (56\%), personal social $(57 \%)$ y desarrollo integral (62\%); en la segunda evaluación también existió una estimación media, pero con incremento en sus porcentajes; además, se evidencia mejoras en cuanto al desarrollo socioafectivo, lingüístico y capacidades cognitivas. Se concluye, que un adecuado uso e implementación de programas de estimulación temprana, garantizan el fortalecimiento de actividades cognitivas, ayudando a la estabilidad emocional del niño para desenvolverse en la sociedad.

Palabras clave: Desarrollo cognitivo; estimulación temprana; neuroeducación; desarrollo socioafectivo; destrezas lingüísticas.

Magister en Educación Inicial. Licenciada en Ciencias de la Educación mención Educación Parvularia y Básica Inicial. Docente de Educación en la Universidad Estatal de Bolívar, Ecuador. E-mail: mcoello@ueb.edu.ec iD ORCID: https://orcid.org/0000-0002-4139-4064 


\title{
Early stimulation and development of language skills: Neuroeducation in initial education in Ecuador
}

\begin{abstract}
Early stimulation is considered a fundamental factor to develop and enhance the child's psychomotor brain functions, helping to strengthen cognitive, linguistic, motor and social aspects. The objective of the research is to analyze the neurological and theoretical aspects that underpin the early stimulation program in early childhood education children in Ecuador. The study is mixed, using descriptive and interpretive techniques with phenomenological, field and correlational design. The population consisted of 400 children of initial education I and II of Educational Units of the province of Bolívar-Ecuador, using the Ortiz Abbreviated Development Scale as an instrument to know linguistic abilities and skills from the neuroeducation approach. The results indicate that, in the first evaluation, an average estimate is maintained, gross motor skills (56\%), adaptive fine motor skills ( $71 \%$ ), hearing and language $(56 \%)$, social personal $(57 \%)$ and integral development $(62 \%)$; In the second evaluation there was also an average estimate, but with an increase in its percentages; In addition, improvements are evidenced in terms of socio-affective, linguistic development and cognitive abilities. It is concluded that an adequate use and implementation of early stimulation programs guarantee the strengthening of cognitive activities, helping the emotional stability of the child to function in society.
\end{abstract}

Keywords: Cognitive development; early stimulation; neuroeducation; socio-affective development; language skills.

\section{Introducción}

La estimulación temprana es considerada como un programa conducido al fortalecimiento de las capacidades cognitivas, socioafectivas, lingüísticas y psicomotrices en niños de edades comprendidas entre los cero y seis años. Son actividades neurálgicas que se fundamenta en la importancia de la neurociencia y neuroeducación para aprovechar los aprendizajes psicológicos, pediátricos y en general las ciencias de la educación. Tiene consigo un proceso de integración que permite el buen desenvolvimiento de posturas diversas que van desde lo físico y deportivo, sin perder de vista los juegos y las actividades artísticas, así como musicales. Al respecto, Guerra, Zuluaga y Saravia (2019) sostienen que "la música es un factor relevante en la vida de las personas, cuyos pensamientos están predispuestos a niveles de creación, crítica y actuaciones elevadas" (p.62).
La familia es determinante para que el programa sea efectivo, sobre todo la parte socio-afectiva, así como el apoyo de acciones gubernamentales y del sector privado. Para lograr este acercamiento, es necesario entender que este proceso resulta ser multidisciplinario, y que se conozcan aspectos técnicos, educativos, socioeconómicos; que estimulen el desarrollo cognitivo y fortalezcan las habilidades, así como destrezas lingüísticas en los niños. La estimulación no es exclusividad para niños normales, también se emplea para mejorar notoriamente las capacidades psicomotrices en aquellos niños que tienen necesidades de educación especial, con discapacidades o disfunción auditiva y visual.

La importancia del desarrollo psicomotriz en los niños surge porque el proceso de expansión que se genera en el cerebro en los primeros cinco años de vida, generalmente se caracterizan por tener un alto grado de plasticidad neuronal, que permiten 
la activación y generación de funciones esenciales, como, por ejemplo: Control postural, aprendizaje cognitivo y el lenguaje (Guillen et al. 2018).

En relación con esto último, Jami et al. (2020) argumentan que es necesario concientizar y asumir que los avances se generan de forma paulatina y que los resultados no se harán visibles de manera inmediata, dicho de otra manera, los avances y desarrollos se manifiestan a mediano plazo, mientras que otros resultados son de tipo observables después de un tiempo prolongado.

Es precisamente a partir de este razonamiento, que surge la actividad denominada estimulación temprana, que según Véliz (2019), se refiere a toda actividad dirigida a niños desde cero a seis años de edad con el propósito de fortalecer y desarrollar de manera oportuna su potencial psicomotriz. Por su parte, Velásquez (2016) señala que la estimulación temprana logra impulsar el desarrollo psicomotor de los niños, permitiendo la evolución cognitiva mediante actividades que van directas al aprendizaje futuro. En este mismo orden de ideas, Barreno y Macías (2015) resaltan que la estimulación busca alentar el talento innato que tienen los pequeños para aprender con facilidad, teniendo en cuenta los períodos sensitivos, lingüísticos y cognitivos.

En este sentido, la estimulación temprana es una de las actividades implementadas en los niños para desarrollar su intelecto. Por lo tanto, la palabra correcta que debería emplearse en el proceso de estimulación temprana sería motivación, debido a que por medio del estímulo aprenden, adquieren seguridad y confianza (Diamond y Ling, 2016).

En otro orden de ideas, resulta oportuno expresar que el desarrollo del lenguaje se adhiere al comportamiento y crecimiento de la madurez cerebral del niño, así como a la integración socio-familiar en el que se desenvuelve (Rodríguez, Orozco y Rodríguez, 2016). En ese sentido, Terranova et al. (2019) manifiestan que, el desarrollo cognitivo se relaciona "con la práctica en la crianza familiar, la manera como la dinámica familiar se autorregulan, resuelven los problemas y establecen mecanismos asertivos de comunicación" (p.332).

El lenguaje infantil se inicia con un período pre-lingüístico donde el menor produce cortos balbuceos que con el tiempo se convertirán en expresiones comunicativas, así como sonoras para poder conectarse con su exterior. Aproximadamente, a partir del octavo mes, el balbuceo acciona un comportamiento ecolálico, es decir, existe una perturbación del lenguaje del que posteriormente surgirán sonidos primarios tales como mamá o papá, y es en esta etapa donde comienza el proceso evolutivo de producir nuevas palabras (Bonilla et al. 2019). Esta transformación sonora del lenguaje por lo general es más lenta que el de comprensión, pero a su vez, resulta suficientemente eficaz cuando se va enriqueciendo el número de palabras y el significado en el mundo exterior (Avendaño et al. 2020).

Lo anteriormente expuesto, ha sido explicado en el tiempo desde diferentes posturas epistemológicas, influyendo las épocas, costumbres y modernismo; tomando en consideración la adquisición del lenguaje desde un punto de vista evolutivo y/o pedagógico. Para comprender la relevancia del lenguaje dentro de la estimulación temprana, a continuación, se detallan someramente las principales teorías desarrolladas que sustentan la relación:

a. La teoría constructivista: Bruner (1995), sugiere que cuando se enfrenta con un sistema nuevo lingüístico, es importante mantener la progresión iniciando con la representación que va desde lo icónico hasta lo simbólico, válido para cualquier estudiante sin importar su edad (Mensah, 2015).

b. Teoría cognitiva del desarrollo del lenguaje, propuesta por Piaget (1984) quién teorizó que el lenguaje se compone de símbolos y estructuras que se van madurando en conjunto con las habilidades mentales del niño (Dastpak, Behjat y Taghinezhad, 2017).

c. Teoría sociocultural, impulsada por Vygotsky (1995), observa al desarrollo humano como un proceso mediado 
socialmente en el que los niños adquieren sus valores culturales, creencias y estrategias de resolución de adversidades, a través de diálogos colaborativos con miembros de la sociedad que se encuentran mejor informados que ellos (Bozkurt, 2017).

Una vez expresadas estas teorías, es oportuno describir la importancia del lenguaje y su función dentro del proceso neurológico; las funciones del lenguaje incluyen la comunicación, la expresión de identidad, el juego, la expresión imaginativa y la liberación emocional (Pinos, Ayala y Bonilla, 2018). Bajo esta misma perspectiva, Martínez, Quintero y Ruíz (2013) lo definen como un sistema formal que incluye sonidos, signos, símbolos $\mathrm{y}$ gestos que se utilizan como un medio importante para comunicar los pensamientos, ideas y emociones de un individuo a otro. En este sentido, el objetivo de la presente investigación consiste en analizar los aspectos neurológicos y teóricos que fundamentan el programa de estimulación temprana en niños de educación inicial en Ecuador.

\section{Neuroeducación}

El siguiente punto trata de la neurociencia $y$, en este sentido, Uscategui y Boscan (2017) expresan que el objetivo es optimizar los procesos de enseñanza y aprendizaje, tomando en consideración los saberes sobre el funcionamiento del cerebro humano. Mora (2017), la refiere como aquella disciplina que brinda un camino al conocimiento para revelar complejidades. Este conocimiento resalta la calidad, que, según Bonilla, Guerrero y López (2018) lo considera indicativo de un alto nivel de satisfacción aunado con la urgencia de internalizar nuevas concepciones, que se encuentran atadas a un proceso psicológico y que son aplicadas para resolver situaciones extremas.

Así pues, Nizama (2015) denota que mediante la neurociencia se puede estudiar el sistema nervioso sobre todo el desarrollo psicomotor y las estructuras cognoscitivas. Es preciso destacar, que el cerebro, el cual es conocido como el órgano central del sistema nervioso, persigue y analiza una serie de patrones que permiten decidir ciertos pensamientos (Flórez, 2015). En este propósito, los hallazgos encontrados en estudios se han aplicado en áreas como: Neurociencia cognitiva, neurociencia afectiva, neuro-estética y neuro-economía, con la intención de buscar correlacionar patrones de conductas de activación y funcionamiento de las neuronas.

En tal sentido, la neurociencia conglomera un conjunto de conocimientos que involucra a la psicología, sociología y medicina, así como también, detecta todas aquellas necesidades motoras como leer, escribir o aprender. Las cuales pueden tener origen en alteraciones cognoscitivas, produciendo entornos estresantes, negativos $\mathrm{y}$ en permanente amenaza e incertidumbre (Salas, 2003). Para indagar de manera científica las bondades de la neurociencia, existen técnicas y herramientas de medición que se utilizan, para conocer y potencializar toda su importancia motora. Entre esas técnicas se destacan: a) $\mathrm{La}$ resonancia magnética, b) la electroencefalografía, c) la optogenética, d) seguimiento visual, e) estudio de las expresiones psico-faciales, f) actividad electro-dérmica, y g) la reacción (Friedrich y Preiss, 2003; Nizama, 2015).

Todas estas técnicas, conducen a adentrarse en el círculo que rodea a la neurociencia, en el cual destacan temáticas interesantes que consolida de forma transversal el mundo de la neurología, la psicología, la estimulación temprana, y la investigación educativa; quienes impulsan lo que se conoce como la neuroeducación (Bravo, 2015). Para Bowers (2016), la importancia de la neurociencia se direcciona hacia la educación para desarrollar los procesos cognoscitivos en los seres humanos. La neuroeducación, es considerada por Bruer (2016) como la nueva interdisciplina, cuyo énfasis radica en la integración de ciencias relacionadas con el aprendizaje y el desarrollo neurocognitivo del ser humano.

El término neuroeducación, Battro 
(2000) lo asemeja con integración e interacción entre las ciencias de la educación y las neurociencias, todas asociadas a la investigación detallada del comportamiento del cerebro y del poder de la mente. Algo interesante que resaltar, es que estando en la búsqueda de modelos adaptables a la neuroeducación, conviene prestar atención a la agenda científica, puesto que no todo lo que se puede hacer, siempre no es lo correcto. Es importante respetar los valores, la ética de los métodos neurobiológicos que están ligados al sistema de enseñanza y aprendizaje (Illes, 2005).

\section{Metodología}

La investigación fue de tipo mixta (cuantitativo y cualitativo), se empleó técnicas descriptivas e interpretativas haciendo énfasis hacia el diseño fenomenológico, así como el análisis numérico de datos estadísticos basados en herramientas de estadística descriptiva e inferencial. Dentro de la parte cuantitativa, se contempló el uso del diseño no experimental transversal, el cual permitió analizar e interpretar los datos por medio de instrumentos de investigación. El estudio fue de campo, sustentado en lo expresado por HernándezSampieri y Mendoza (2018), quienes lo conceptúan como un “(...) experimento efectuado en una situación más real o natural en la que el investigador manipula una o más variables independientes" (p.172). En esta modalidad los investigadores toman contacto en forma directa con la realidad, para obtener información de acuerdo con los objetivos propuestos.

El nivel de la investigación, fue correlacional, el cual Hernández et al. (2017) expresan que “(...) se emprenderá estudios correlacionales para conocer la relación o grado de asociación entre dos o más conceptos, categorías o variables en determinado contexto" (p.77). La finalidad fue conocer el grado de asociación que existe entre los conceptos y teorías en el estudio. A su vez, mide la importancia entre las variables, utilizando coeficientes de correlación estadísticos, es decir, miden cada variable presuntamente relacionada y después calculan, así como analizan su correlación.

Para desarrollar la correlación, se tomó en consideración la hermenéutica, cuyo propósito es analizar los puntos teóricos más pertinentes y cohesionarlos con los ejes temáticos, desde distintas perspectivas para consolidarla en una sola opinión. La población y muestra de estudio fue seleccionada bajo un muestreo no probabilístico por conveniencia, por ello, se trabajó con 400 niños de educación inicial I y II de aquellas Unidades Educativas situadas en la provincia de Bolívar, cantón de Guaranda en Ecuador.

Se empleó la Escala Abreviada de Desarrollo de Ortiz (EAD), que consiste en una revisión científica enfocada en la conceptualización para realizar la selección de indicadores, centrándose en las habilidades y destrezas del lenguaje desde el enfoque de la neuroeducación en niños de educación inicial, en las Unidades Educativas, de la provincia Bolívar, cantón Guaranda, Ecuador. La confiabilidad de la escala por áreas es: Motricidad gruesa (92\%), Motricidad fina adaptativa (93\%), Audición y Lenguaje (91\%) y Personal Social (91\%), así como la validez es de $0.73 \%$ del desarrollo total de la prueba.

Los items se organizaron basándose en la observación y recopilación de datos teóricos como numéricos en un ambiente pedagógico y psicológico. La aplicación del instrumento permitió detectar casos de alto riesgo de retardo, los criterios facilitaron la ubicación de los indicadores en cada rango de edad maximizando la posibilidad de identificación de niños con dificultades en el lenguaje.

Para determinar la importancia de la estimulación temprana en los niños evaluados, en primer lugar, se procedió a emplear el instrumento EAD antes de comenzar a aplicar los ejercicios previamente seleccionados. $\mathrm{La}$ intención es tener datos primarios (Educación Inicial - antes) que indiquen los niveles psicomotrices, cognoscitivos y lingüísticos iniciales de cada niño antes de comenzar las secciones, para posteriormente, 
poder compararlas con la segunda medición (Educación Inicial - después). En un lapso prudencial y luego de haber entendido todo el proceso de lo que significa la estimulación, y logrando integrar a los padres, en especial a las madres dentro de las actividades, por segunda ocasión se procedió a emplear nuevamente el instrumento, para obtener nuevos datos (secundarios) y observar, analizar, así como evaluar los efectos, avances o retrocesos productos de la actividad estimuladora en los niños.

Con el fin de poder analizar los resultados de la aplicación del instrumento, fue importante conocer los materiales que intervienen en el proceso. El recurso humano es uno de ellos, y más aún cuando se toman en consideración la evaluación sobre cinco aspectos fundamentales: Motricidad gruesa, motricidad fina adaptativa, audición $\mathrm{y}$ lenguaje, personalidad social y desarrollo integral. Los especialistas (educadores), se convirtieron en el principal objeto material que se utilizó mediante la evaluación.

También intervinieron otros materiales como sonajas (maracas), cronómetros, cinta métrica, pelota, escalerillas, linterna, cubos, juguetes llamativos, libros de cuentos, cucharas de plástico, hojas de papel, creyones, entre otros; que permitieron al especialista observar el comportamiento del niño y evaluar en los respectivos renglones de los diferentes niveles del instrumento para generar datos que tienen que ver con el estatus generalizado cognitivo del niño evaluado.

Las repeticiones fueron importantes durante este proceso, es allí donde los padres jugaron un papel relevante porque deben conocer las distintas técnicas existentes de cómo se manejan estos ejercicios, para poder repetirlos en el hogar. A medida que se refuerzan las actividades, los resultados pueden acelerarse y el nivel del niño se irá fortaleciendo haciéndose más seguro y teniendo mayor confianza.

Para interpretar los resultados se empleó un lenguaje sumamente sencillo, claro y ceñido al nivel de comprensión de los involucrados, asegurando que la información pudiera ser entendida y evitar confusiones, así como erróneas interpretaciones inherente a la medición, que variara los puntos de vistas y trayectorias individuales en el desarrollo de las actividades de estimulación temprana en los niños, dentro de la escala para captar de manera concreta las capacidades cognitivas. Se procuró que el lenguaje fuera propositivo y que movilizara la sensibilidad del cuidador, garantizando la atención que requería las condiciones de cada pequeño.

\section{Resultados y discusión}

Dentro de este apartado, se muestran los resultados, luego de haber aplicado la fase I (Educación Inicial - antes) para determinar los valores iniciales de los niños evaluados y poder compararlos más adelante (fase IIEducación Inicial- después), una vez aplicadas las respectivas actividades relacionadas con la estimulación temprana, en concordancia con la participación integral de la familia del pequeño, como parte del compromiso de reforzamiento en casa.

\subsection{Fase I: Educación inicial - Pre- Test (antes)}

En la valoración de motricidad gruesa se evidencia en la Tabla 1, casi tres cuartos de la población evaluada presentan una estimación de categoría media, valoración que se debe considerar puesto que representa el equilibrio mínimo neural y psicomotriz. Por otra parte, más de un cuarto $(39 \%)$ de la población se encuentra en la categoría alerta. En lo que se refiere a la estimación de medio alto, hay una minina presencia puesto que menos de la mitad de un cuarto de la población se encuentra en dicha valoración. Esto indica que se deben tomar todas las previsiones para enfrentar esta parte media de la población. En consecuencia, los programas de estimulación temprana deben abarcar desarrollos de destreza y habilidades para evitar que los alumnos lleguen a niveles superiores. 
Tabla 1

Motricidad gruesa

\begin{tabular}{lllllll}
\hline Estimación & Frecuencia & Porcentaje & Media & Mediana & $\begin{array}{l}\text { Desviación } \\
\text { estándar }\end{array}$ & Varianza \\
\hline Alerta & 158 & $39,5 \%$ & & & & \\
Medio & 224 & $56,0 \%$ & & & & \\
Medio alto & 18 & $4,5 \%$ & 1.65 & 2.00 & .564 & .318 \\
Alto & 0 & $0,0 \%$ & & & & \\
Total & $\mathbf{4 0 0}$ & $\mathbf{1 0 0 , 0 \%}$ & & & & \\
\hline
\end{tabular}

Fuente: Elaboración propia, 2020.

En los estudiantes se valoró la dimensión de motricidad fina adaptativa, como se plantea en la Tabla 2, cerca de tres cuartos de la población (71\%) mantienen una valoración cualitativa medio. Por otra parte, aproximadamente un cuarto de los escolares evaluados mantiene una valoración de alerta, así como, cerca de la mitad de un cuarto de estudiantes ha desarrollado un nivel medio alto. Finalmente, es importante destacar, que tan solo una mínima parte de la población registran un desarrollo alto de motricidad fina adaptativa $(2,5 \%)$.

Tabla 2

Motricidad fina adaptativa

\begin{tabular}{lcrrrrr} 
Estimación & Frecuencia & Porcentaje & Media & Mediana & $\begin{array}{c}\text { Desviación } \\
\text { estándar }\end{array}$ & Varianza \\
\hline Alerta & 76 & $19,0 \%$ & & & & .362 \\
Medio & 284 & $71,0 \%$ & & & \\
Medio alto & 30 & $7,5 \%$ & 1.94 & 2.00 & & .362 \\
Alto & 10 & $2,5 \%$ & & & \\
Total & $\mathbf{4 0 0}$ & $\mathbf{1 0 0 , 0} \%$ & & & \\
\hline
\end{tabular}

Fuente: Elaboración propia, 2020.

Estos resultados son consecuencias de lo mostrado en la Tabla 1, por lo tanto, se deben clasificar los alumnos de acuerdo a su edad y estado motriz. De esta forma, los niños avanzados seguirán por rumbos efectivos evitando cualquier retraso lingüístico que pudiera haber sido evaluado y tratado con anterioridad. Evitar en lo posible retrocesos en el desarrollo de las actividades cognitivas.

Para conocer el desarrollo de habilidades y destrezas del lenguaje en los niños, se ha evaluado su dimensión y alcance. Considerando los resultados de la dimensión de audición y lenguaje se evidencia en la Tabla 3, que por encima de dos cuartos de los escolares evaluados registran una estimación media (56\%), asimismo, un poco más de un cuarto de la población se ubica en el nivel de valoración de alerta. Finalmente, cerca de la mitad de un cuarto de estudiantes evaluados presentan un nivel medio alto dentro de la dimensión puesta en consideración. 
Tabla 3

Audición y Lenguaje

\begin{tabular}{lcrrrrr}
\hline Estimación & Frecuencia & Porcentaje & Media & Mediana & $\begin{array}{c}\text { Desviación } \\
\text { estándar }\end{array}$ & Varianza \\
\hline Alerta & 134 & $33,5 \%$ & & & & \\
Medio & 224 & $56,0 \%$ & 1.77 & 2.00 & .623 & .388 \\
Medio alto & 42 & $10,5 \%$ & & & \\
Alto & 0 & $0,0 \%$ & & & \\
Total & $\mathbf{4 0 0}$ & $\mathbf{1 0 0 , 0 \%}$ & & & \\
\hline
\end{tabular}

Fuente: Elaboración propia, 2020.

En la Tabla 4, se encuentra la valoración de la dimensión personal social, se evidencia que un poco más de dos cuartos $(57,5 \%)$ se encuentran bajo una estimación medio en el desarrollo de habilidades sociales, por otro lado, menos de dos cuartos de los niños evaluados cuenta con un desarrollo en categoría alerta y, menos de la mitad de un cuarto se encuentra en la categoría de medio alto. En esta dimensión, también se registra un porcentaje mínimo de estudiantes que mantienen una estimación alta (1\%). Estos datos indican que uno de los objetivos de la investigación es el desarrollo de las habilidades y destrezas lingüísticas en niños que se encuentran en la educación inicial, por lo tanto, su incursión a la sociedad debe ser prioridad. Todos los programas de desarrollo de la estimulación temprana deben estar enmarcados a que el niño puede soportar todas estas cargas sociales, utilizando herramientas de lenguaje que permita comunicarse con su entorno.

Tabla 4

Personal social

\begin{tabular}{|c|c|c|c|c|c|c|}
\hline Estimación & Frecuencia & Porcentaje & Media & Mediana & $\begin{array}{l}\text { Desviación } \\
\text { estándar }\end{array}$ & Varianza \\
\hline Alerta & 154 & $38,5 \%$ & \multirow{5}{*}{1.66} & \multirow{5}{*}{2.00} & \multirow{5}{*}{.554} & \multirow{5}{*}{.307} \\
\hline Medio & 230 & $57,5 \%$ & & & & \\
\hline Medio alto & 12 & $3,0 \%$ & & & & \\
\hline Alto & 4 & $1,0 \%$ & & & & \\
\hline Total & 400 & $100,0 \%$ & & & & \\
\hline
\end{tabular}

Fuente: Elaboración propia, 2020.

Mediante la aplicación de la Escala Abreviada de Desarrollo de Ortiz, y posterior a las evaluaciones por dimensiones, se obtiene como resultado global que cerca de tres cuartos de los alumnos escolares $(62 \%)$ poseen un desarrollo integral medio alto, un poco más de un cuarto de estudiantes presentan un desarrollo integral medio (28\%). De igual forma, aproximadamente casi la mitad de un cuarto de los niños evaluados se encuentran en alerta con su desarrollo integral. 
Tabla 5

Desarrollo integral

\begin{tabular}{lcccccc}
\multicolumn{1}{c}{ Estimación } & Frecuencia & Porcentaje & Media & Mediana & $\begin{array}{c}\text { Desviación } \\
\text { estándar }\end{array}$ & Varianza \\
\hline Alerta & 34 & $8,5 \%$ & & & & .419 \\
Medio & 112 & $28,0 \%$ & 2.55 & 2.00 & .647 & \\
Medio alto & 250 & $62,5 \%$ & & & \\
Alto & 4 & $1,0 \%$ & & & \\
Total & $\mathbf{4 0 0}$ & $\mathbf{1 0 0 , 0 \%}$ & & & \\
\hline
\end{tabular}

Fuente: Elaboración propia, 2020.

Esta alerta (8,5\%) significa que se deben analizar los datos y adaptar los procesos en la medida que los resultados se originen. Por consiguiente, el diseño y rediseño de los programas resultará lo más importante durante la sistematización de los mismos. Finalmente, el $1 \%$ restante de los niños evaluados presentan un desarrollo integral alto (ver Tabla 5).

En la valoración de los escolares se buscó conocer la relación entre las dimensiones evaluadas. Para tal cometido, se aplicó una prueba no paramétrica conocida como Correlación de Rho de Spearman, la cual trabaja con variables de tipo ordinal (ver Tabla 6). Además, el estadístico tiene la finalidad de cuantificar la correlación de dichas variables. Por lo descrito anteriormente, el coeficiente de mayor valor y, por ende, el que muestra mayor correlación, es el obtenido entre la variable audición y lenguaje con la variable motricidad fina adaptativa (.780) evidenciando así una correlación positiva considerable.

Tabla 6

Correlación de dimensiones evaluadas

\begin{tabular}{|c|c|c|c|c|c|c|}
\hline & & $\begin{array}{c}\text { Motricidad } \\
\text { gruesa }\end{array}$ & $\begin{array}{c}\text { Motricidad fina } \\
\text { adaptativa }\end{array}$ & $\begin{array}{c}\text { Audición y } \\
\text { lenguaje }\end{array}$ & $\begin{array}{c}\text { Personal } \\
\text { social }\end{array}$ & Total \\
\hline $\begin{array}{l}\text { Motricidad } \\
\text { gruesa }\end{array}$ & $\begin{array}{l}\text { Coeficiente de } \\
\text { correlación }\end{array}$ & 1.000 & $.645^{* *}$ & $.752^{* * *}$ & $.706^{* * *}$ & $.752^{* *}$ \\
\hline $\begin{array}{l}\text { Motricidad fina } \\
\text { adaptativa }\end{array}$ & $\begin{array}{l}\text { Coeficiente de } \\
\text { correlación }\end{array}$ & $.645^{* *}$ & 1.000 & $.780^{* * *}$ & $.665^{* * *}$ & $.657^{* *}$ \\
\hline $\begin{array}{l}\text { Audición y } \\
\text { lenguaje }\end{array}$ & $\begin{array}{l}\text { Coeficiente de } \\
\text { correlación }\end{array}$ & $.752^{* *}$ & $.780^{* * *}$ & 1.000 & $.760^{* * *}$ & $.771^{* * *}$ \\
\hline Personal social & $\begin{array}{l}\text { Coeficiente de } \\
\text { correlación }\end{array}$ & $.706^{* *}$ & $.665^{* *}$ & $.760^{* * *}$ & 1.000 & $.656^{* *}$ \\
\hline \multirow{2}{*}{ Total } & $\begin{array}{l}\text { Coeficiente de } \\
\text { correlación }\end{array}$ & $.752^{* * *}$ & $.657^{* * *}$ & $.771^{* * *}$ & $.656^{* * *}$ & 1.000 \\
\hline & $\mathrm{N}$ & 400 & 400 & 400 & 400 & 400 \\
\hline
\end{tabular}

Nota: **. La correlación es significativa en el nivel 0.01 (bilateral)

Fuente. Elaboración propia, 2020.

Por otra parte, entre la variable audición y lenguaje, en conjunto con la variable relacionada con la motricidad gruesa, también tiene una correlación positiva (.752). La variable personal social y motricidad (.665); no existiendo ningún tipo de distanciamiento, más bien, observándose consistencia en los resultados, lo que permite indagar que el instrumento fue empleado de manera correcta. 


\subsection{Educación Inicial - Post Test (después)}

Se observa que en los estudiantes de educación inicial cerca de tres cuartos de la población (61\%) mantiene un desarrollo medio de la habilidad (ver Tabla 7); mientras que, por encima de un cuarto de la población se encuentra en la estimación de alerta. Por otra parte, aproximadamente, la mitad de un cuarto de la muestra $(7,5 \%)$ de la investigación mantiene un desarrollo medio alto, asimismo, se observa que una mínima parte de escolares evaluados mantiene un nivel alto $(1 \%)$ dentro de la evaluación realizada. Las frecuencias demuestran que, a pesar de ser de un nivel superior a los resultados anteriores, la motricidad es el eje central que permite evaluar el alcance y el direccionamiento de los programas.

Tabla 7

Motricidad gruesa

\begin{tabular}{lcrcccc}
\hline Estimación & Frecuencia & Porcentaje & Media & Mediana & Desviación estándar & Varianza \\
\hline Alerta & 122 & $30,5 \%$ & & & & .377 \\
Medio & 244 & $61,0 \%$ & 1.79 & 2.00 & & .614 \\
Medio alto & 30 & $7,5 \%$ & & & & \\
Alto & 4 & $1,0 \%$ & & & & \\
Total & $\mathbf{4 0 0}$ & $\mathbf{1 0 0 , 0 \%}$ & & & & \\
\hline
\end{tabular}

Fuente: Elaboración propia, 2020.

En la valoración de motricidad fina adaptativa de la población evaluada, en proximidad a tres cuartos de evaluados se ubican en un nivel medio (ver Tabla 8); por otra parte, un cuarto y medio de escolares cuentan con una estimación de alerta. No obstante, con representaciones mínimas, el nivel medio alto se encuentra en un $5 \%$ y el nivel alto únicamente registra tan solo el $2 \%$ aspectos que se deben considerar en la población.

Tabla 8

Motricidad fina adaptativa

\begin{tabular}{lcrrrrr}
\hline Estimación & Frecuencia & Porcentaje & Media & Mediana & $\begin{array}{c}\text { Desviación } \\
\text { estándar }\end{array}$ & Varianza \\
\hline Alerta & 140 & $35,0 \%$ & & & & .613 \\
Medio & 232 & $58,0 \%$ & 1.74 & 2.00 & .643 &. \\
Medio alto & 20 & $5,0 \%$ & & & & \\
Alto & 8 & $2,0 \%$ & & & & \\
Total & $\mathbf{4 0 0}$ & $\mathbf{1 0 0 , 0 \%}$ & & & \\
\hline
\end{tabular}

Fuente: Elaboración propia, 2020.

Considerando los resultados de la dimensión de audición y lenguaje, en la Tabla 9 se evidencia que casi dos cuartos de la población se ubican en el nivel de valoración de alerta (42\%) y nivel medio (47\%), respectivamente; sin embargo, casi la mitad de un cuarto de la población se encuentra valorada en un nivel medio alto, considerando también que únicamente una mínima parte de escolares se encuentran en un nivel alto (1\%). 
Tabla 9

Audición y lenguaje

\begin{tabular}{lcrrrrr}
\hline \multicolumn{1}{c}{ Estimación } & Frecuencia & Porcentaje & Media & Mediana & $\begin{array}{c}\text { Desviación } \\
\text { estándar }\end{array}$ & Varianza \\
\hline Alerta & 168 & $42,0 \%$ & & & & \\
Medio & 188 & $47,0 \%$ & 1.70 & 2.00 & .686 & .471 \\
Medio alto & 40 & $10,0 \%$ & & & & \\
Alto & 4 & $1,0 \%$ & & & & \\
Total & $\mathbf{4 0 0}$ & $\mathbf{1 0 0 , 0 \%}$ & & & \\
\hline
\end{tabular}

Fuente: Elaboración propia, 2020.

Con el fin de conocer la interacción social que desarrollan los estudiantes de educación inicial, se observa que casi la tercera parte de las evaluados se encuentran en un nivel medio $(60 \%)$, por encima de un cuarto de escolares se ubican en un nivel de alerta, asimismo, dentro del rango medio alto, la mitad de un cuarto de la población se encuentran dentro de dicha estimación (ver Tabla 10). Finalmente, en la categorización del nivel alto existe un porcentaje mínimo de estudiantes con esta valoración (2\%). Como se acoto anteriormente, la incursión de los niños a la sociedad, debe ser uno de los objetivos centrales de la investigación. Aplicar la estimulación temprana conlleva a analizar que el desarrollo cognoscitivo de alguna manera sí activa los procesos de motricidad orientados hacia las destreza y habilidades lingüísticas. La comunicación efectiva es la consecuencia de emplear bien estos programas.

Tabla 10

\section{Personal social}

\begin{tabular}{lcrrrrr}
\hline Estimación & Frecuencia & Porcentaje & Media & Mediana & $\begin{array}{c}\text { Desviación } \\
\text { estándar }\end{array}$ & Varianza \\
\hline Alerta & 126 & $31,5 \%$ & & & & .417 \\
Medio & 240 & $60,0 \%$ & 1.79 & 2.00 & .646 & \\
Medio alto & 26 & $6,5 \%$ & & & \\
Alto & 8 & $2,0 \%$ & & & \\
Total & $\mathbf{4 0 0}$ & $\mathbf{1 0 0 , 0 \%}$ & & & \\
\hline
\end{tabular}

Fuente: Elaboración propia, 2020.

Al finalizar la valoración de las dimensiones en los estudiantes de inicial II ,se obtiene el siguiente resultado final (ver Tabla 11), donde dos cuartos de la población evaluada mantienen un nivel medio (50\%). Un poco más de un cuarto de escolares mantiene un nivel de alerta $(29,5 \%)$. Adicional a ello, cerca de un cuarto de niños/as evaluados registran un nivel medio alto, y finalmente, tan solo una mínima parte de la población presenta una valoración de desarrollo alto (2\%). 
Tabla 11

Desarrollo integral

\begin{tabular}{lcccccc}
\hline Estimación & Frecuencia & Porcentaje & Media & Mediana & $\begin{array}{c}\text { Desviación } \\
\text { estándar }\end{array}$ & Varianza \\
\hline Alerta & 118 & $29,5 \%$ & & & & .746 \\
Medio & 200 & $50,0 \%$ & 1.93 & 2.00 & & .556 \\
Medio alto & 74 & $18,5 \%$ & & & \\
Alto & 8 & $2,0 \%$ & & & \\
Total & $\mathbf{4 0 0}$ & $\mathbf{1 0 0 , 0} \%$ & & & \\
\hline
\end{tabular}

Fuente: Elaboración propia, 2020.

En la valoración de los escolares se buscó conocer la relación entre las dimensiones evaluadas. Para tal cometido, se aplicó una prueba no paramétrica conocida como Correlación de Rho de Spearman, la cual trabaja con variables de tipo ordinal; además, el estadístico tiene la finalidad de cuantificar la correlación de dichas variables. Por lo descrito anteriormente, se la Tabla 12 se evidencia que el coeficiente de mayor valor $\mathrm{y}$, por ende, el que muestra mayor correlación, es el obtenido entre la variable motricidad gruesa con la variable personal social (.983), evidenciando así una correlación positiva considerable. Por otra parte, entre la variable motricidad fina auditiva, en conjunto con la variable relacionada con la personal social, también tiene una correlación positiva (.931).

Tabla 12

Correlación de dimensiones evaluadas

\begin{tabular}{|c|c|c|c|c|c|c|}
\hline & & $\begin{array}{c}\text { Motricidad } \\
\text { gruesa }\end{array}$ & $\begin{array}{l}\text { Motricidad } \\
\text { fina } \\
\text { adaptativa }\end{array}$ & $\begin{array}{l}\text { Audición } \\
\text { y lenguaje }\end{array}$ & $\begin{array}{l}\text { Personal } \\
\text { social }\end{array}$ & Total \\
\hline Motricidad gruesa & $\begin{array}{l}\text { Coeficiente de } \\
\text { correlación }\end{array}$ & 1.000 & $.915^{* *}$ & $.824^{* *}$ & $.983^{* *}$ & $.902^{* *}$ \\
\hline $\begin{array}{l}\text { Motricidad fina } \\
\text { adaptativa }\end{array}$ & $\begin{array}{l}\text { Coeficiente de } \\
\text { correlación }\end{array}$ & $.91^{5^{*} *}$ & 1.000 & $.871^{* *}$ & $.931^{* *}$ & $.850^{* *}$ \\
\hline Audición y lenguaje & $\begin{array}{l}\text { Coeficiente de } \\
\text { correlación }\end{array}$ & $.824^{* * *}$ & $.871^{* *}$ & 1.000 & $.837^{* *}$ & $.819^{* * *}$ \\
\hline Personal social & $\begin{array}{l}\text { Coeficiente de } \\
\text { correlación }\end{array}$ & $.983^{* * *}$ & $.931^{* *}$ & $.837^{* *}$ & 1.000 & $.892^{* * *}$ \\
\hline \multirow[t]{2}{*}{ Total } & $\begin{array}{l}\text { Coeficiente de } \\
\text { correlación }\end{array}$ & $.902^{* * *}$ & $.850^{* *}$ & $.819^{* *}$ & $.892^{* *}$ & 1.000 \\
\hline & $\mathrm{N}$ & 400 & 400 & 400 & 400 & 400 \\
\hline
\end{tabular}

Fuente. Elaboración propia, 2020. 
Finalmente, las variables motricidad fina adaptativa y motricidad gruesa también presenta una correlación considerable pues el valor obtenido se enmarca en .915 (ver Tabla 12). A partir de lo señalado, no se registra ningún tipo de distanciamiento, más bien, se observa una consistencia significativa en los resultados obtenidos, lo que permite determinar estadísticamente que el instrumento fue empleado de manera correcta.

$\mathrm{Si}$ se realiza una comparación entre los resultados de las correlaciones entre dimensiones de educación inicial antes y educación inicial después, considerando los resultados más relevantes, se identifica en la Tabla 13, que en la relación de personal social y desarrollo integral existe una diferencia notoria entre los estudiantes de educación inicial antes y después, en el primer grupo existe una correlación positiva considerable de .656; a pesar de esto, en el segundo grupo evaluado existe una correlación positiva muy fuerte (.885).

\section{Tabla 13}

\section{Comparación de los resultados entre educación inicial antes y educación inicial después}

\begin{tabular}{|c|c|c|c|c|c|c|c|c|c|c|c|c|}
\hline & & & 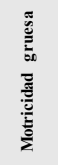 & 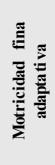 & 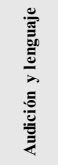 & 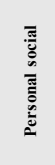 & 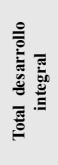 & 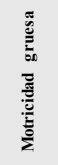 & 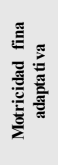 & 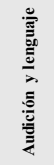 & 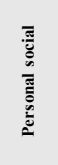 & 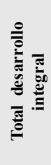 \\
\hline $\begin{array}{l}\text { Motricidad } \\
\text { gruesa }\end{array}$ & $\begin{array}{l}\text { Coeficiente } \\
\text { correlación }\end{array}$ & de & 1.00 & $.645^{* *}$ & $.752^{* *}$ & $.706^{* *}$ & $.752^{* *}$ & 1.00 & $.915^{* *}$ & $.849^{* *}$ & $.976^{* *}$ & $.887^{* *}$ \\
\hline $\begin{array}{l}\text { Motricidad fina } \\
\text { adaptativa }\end{array}$ & $\begin{array}{l}\text { Coeficiente } \\
\text { correlación }\end{array}$ & de & $.645^{* *}$ & 1.00 & $.780^{* *}$ & $.665^{* *}$ & $.657^{* *}$ & $.915^{* *}$ & 1.00 & $.868^{* *}$ & $.943^{* *}$ & $.850^{* *}$ \\
\hline $\begin{array}{l}\text { Audición y } \\
\text { lenguaje }\end{array}$ & $\begin{array}{l}\text { Coeficiente } \\
\text { correlación }\end{array}$ & de & $.752^{* *}$ & $.780^{* *}$ & 1.00 & $.760^{* *}$ & $.771^{* *}$ & $.849^{* *}$ & $.868^{* *}$ & 1.00 & $.853^{* *}$ & $.830^{* *}$ \\
\hline Personal social & $\begin{array}{l}\text { Coeficiente } \\
\text { correlación }\end{array}$ & de & $.706^{* *}$ & $.665^{* *}$ & $.760^{* *}$ & 1.00 & $.656^{* *}$ & $.976^{* *}$ & $.943^{* *}$ & $.853^{* *}$ & 1.00 & $.885^{* *}$ \\
\hline \multirow{2}{*}{$\begin{array}{l}\text { Total desarrollo } \\
\text { integral }\end{array}$} & $\begin{array}{l}\text { Coeficiente } \\
\text { correlación }\end{array}$ & de & $.752^{* *}$ & $.657^{* *}$ & $.771^{* *}$ & $.656^{* *}$ & 1.00 & $.887^{* *}$ & $.850^{* *}$ & $.830^{\circ *}$ & $.885^{* *}$ & 1.00 \\
\hline & $\mathrm{N}$ & & 400 & 400 & 400 & 400 & 400 & 400 & 400 & 400 & 400 & 400 \\
\hline
\end{tabular}

Fuente: Elaboración propia, 2020.

En la relación existente entre audición/ lenguaje y desarrollo integral en el grupo de educación inicial antes, mantienen una correlación positiva considerable (.771); sin embargo, en los escolares de educación inicial después, existe una diferencia relevante debido a que cuentan con una correlación positiva muy fuerte (.830), estimando que el desarrollo de las destrezas y habilidad en educación inicial con la segunda evaluación se encuentran más desarrolladas.

A tenor de los hallazgos encontrados, la estimulación temprana es considerada como la fisonomía más relevante en el desenvolvimiento integral de los niños, que permite el fortalecimiento de áreas funcionales neurocerebrales que favorecen el ámbito cognitivo y lingüístico, así como motor y social. Bajo este contexto, un estudio desarrollado por Contreras (2020), coloca en evidencia que la estimulación motriz y educativa pueden alcanzar cambios en los procesos de aprendizaje en niños.

De esta forma, comienzan a formarse conceptualizaciones completamente sustentables sobre la pedagogía experimental, 
empleando métodos secuenciales previamente analizados e integrándolo a actividades físicas, utilizando o empleando a su vez, ayuda con material didáctico que permiten evaluar y medir los diferentes comportamientos cognoscitivos de los niños. Una de las áreas de interés es la lingüística, el cual Ortiz (2005) la considera como una deficiencia en el desarrollo del lenguaje. El enfoque que le da el autor, es hacia las dificultades lingüística reflejada por la posible capacidad intelectual durante la niñez.

Presentar y mantener una inadecuada habilidad del lenguaje, pudiera ser considerada como una formalidad psicomotriz deficiente. Desde la óptica de otros investigadores (Stodolsky y Lesser, 1967; Baratz, 1969; Labov, 1969; Stewart, 1969), esta posición pareciera insostenible debido a la repercusión que poseen las culturas en relación al diseño, solidez interna y riqueza en sus posibilidades de expresión. Desde la visión cultural, Chavarría (1982) expresa que, en el caso de existir una carencia o penuria cultural, va a ir en contra a los detrimentos neurocognitivos que a su vez afecta de manera directa los niveles de aprendizaje e inteligencia en los niños evaluados.

Este estudio previo, permite dos cosas, la primera identificar el estatus del participante y el segundo, conocer sus avances o retrocesos para afinar el estudio que se está prestando. Para lograr de manera efectiva un buen programa que incluya los elementos mencionados (lingüística y cultura), es importante tomar en consideración la relevancia que tiene la neurociencia en el sistema de estimulación temprana (Coelho, 2017). La neurociencia para Bueno (2017), se encarga de estudiar la estructura, función y desarrollo del sistema nervioso, lo que incluye, el cerebro humano.

Sin embargo, Jadán-Guerrero et al. (2020) denotan que el avance que se ha obtenido en el tiempo, permite afirmar que se alcanzan análisis mucho más complejos que abarca comportamientos que se aprenden mediante modelos, ejemplos y aprendizaje visual generados desde el hogar, ámbito de mayor desenvolvimiento, y lugar donde el niño pasa mayor tiempo al comienzo de su vida. Esta situación permite evidenciar que la comprensión, el afecto y la comunicación, juegan un papel preponderante como factores o elementos que se deben aplicar para lograr integración social y que desde el punto de vista de la neurociencia debe aprovecharse para estudiar el comportamiento del cerebro y la conducta humana (Bueno, 2018).

Martínez (2020) plantea que, entender el verdadero funcionamiento del cerebro permite avanzar en temas como la memoria, la emoción, la conducta del ser humano, el pensamiento, los sentimientos, las capacidades intelectuales y creativas, el aprendizaje, la estimulación temprana y la educación de los niños. Recalca el autor, que la neurociencia es la posibilidad que todas las personas tienen de aprender de una forma, siendo de una $u$ otra manera efectiva; no obstante, Salamanca y Sánchez (2018) sugieren que relacionarse de manera directa con el cerebro, implica que el niño reciba la orientación profesional adecuada para evitar futuros trastornos que afectan la vida social y cotidiana. Para mejorar esta situación, sugiere la implementación de la neuroeducación como herramienta cognitiva que sea capaz de inculcar desde el plano educativo las debidas y correctas líneas del comportamiento mediante el estudio detallado de técnicas de estimulación temprana que coadyuve al desarrollo psicomotriz del niño.

Por consiguiente, por medio de la neuroeducación es posible obtener avances significativos siempre y cuando se tengan presente, los análisis de los resultados y estudios previos, es decir, el antes y el después, que permitan el conocimiento general del grupo de trabajo y evitar que algunos niños que se encuentren en niveles avanzados retrocedan (consistencia), además de lograr la integración total de los padres, quienes a la larga representan el motor indispensable para poder llevar a cabo un programa de estimulación exitoso.

Ahora bien, en relación a este tema de los padres, Megías y Lozano (2019), señalan que, si bien el reforzamiento de los ejercicios es clave en el hogar, también hace énfasis 
que si los ejecutantes, es decir, los padres no cuentan con la experticia del caso, los resultados pudieran ser contra producente. Para contrarrestar esta situación, plantea que la comunicación entre los especialistas, es decir, educadores, y los padres debe ser clara, precisa, y argumentando los beneficios que se obtienen a mediano plazo sobre la ejecución efectiva de los ejercicios.

Para finalizar, queda claro que un buen programa de estimulación temprana, tomando en consideración todos los pros y en contra descritos, van a permitir al niño desarrollar su condición cognitiva hacia elementos de motricidad, audición, lingüística, personalidad, socialización y desarrollo integral de forma exitosa.

\section{Conclusiones}

Entre las características relevantes de la investigación, se determinaron diferentes desarrollos intrínsecos en el proceso de estimulación temprana, entre los cuales se pueden nombrar los siguientes: Motor (caracterizada por un incremento en la movilidad del niño); cognoscitivo (el niño aprende a aprender); lingüístico (necesidad para comunicarse y satisfacer actividades que no puede coordinar ni complementar); visual (reconocimiento como medio para darle significancia de conceptos nuevos en su cerebro); auditivo (búsqueda directa de la fuente de sonidos); socioafectivo (proceso de socialización); gustativo (preferencia por degustaciones); y, olfativo (sensibilidad para los olores).

Es importante que, durante este proceso de estimulación temprana, se realicen de forma conjunta un reforzamiento en casa por parte de los padres que agilice el desarrollo del aprendizaje, por esto, es importante que todos conozcan la utilidad y forma de emplear los ejercicios para garantizar los avances del niño. Hay que recordar que la estimulación no solamente es para un grupo selectivo de pequeños, este proceso debe ser accesible a cualquier persona que amerite desarrollar algunos sectores motrices, físicos, psicológicos, entre otros.

Los niveles de desarrollo de habilidades y destrezas lingüísticas, determinadas antes y después de aplicar el programa de estimulación temprana, trajo como consecuencias la identificación de áreas representadas por enfoques físicos (motricidad gruesa y fina adaptativa), cognoscitivos (audición y lingüística) y socioafectivas (socialización del niño), todos de manera conjunta representan el desarrollo integral del sistema que busca sistematizar las habilidades y destrezas enmarcadas dentro de la neurociencia y neuroeducación en los niños cursantes en la etapa inicial.

Estos hallazgos son importantes porque se han podido identificar etapas clave del desarrollo infantil que pueden ayudar a determinar la salud mental de los niños más adelante en sus carreras académicas. $\mathrm{Al}$ comprender que la cantidad de lenguaje a la que un niño está expuesto temprano en la vida es importante, puesto que, se puede crear intervenciones y programas que logran ayudar a los padres y proveedores de cuidado infantil a mejorar la exposición al lenguaje durante esta edad crítica del desarrollo.

Por ello, se recomienda que las futuras líneas investigativas se direccionen a conocer los riesgos y futuras alteraciones. A partir del análisis de los factores de riesgo, se puede conocer el desarrollo del lenguaje infantil, y esta puede influir en las habilidades preverbales hasta la adolescencia, y como consecuencia generar problemas en la edad escolar, así como en general.

\section{Referencias bibliográficas}

Avendaño, P., Hernández-Mosqueira, C., Fernandes, S., Peña, S., PavezAdasme, G., Troyano, A., Castillo, H., y Fernandes, J. (2020). Destrezas motoras y marcadores Genéticos en niños con Trastorno Específico del lenguaje y desarrollo típico del 
lenguaje. Retos, 38, 235-241. https:// doi.org/10.47197/retos.v38i38.71461

Baratz, J. A. (1969). Linguistic and cultural factors in teaching reading to ghetto children. Elementary English, 46(2), 199-203. https://www.jstor.org/ stable/41386487

Barreno, Z., y Macías, J. (2015). Estimulación temprana para potenciar la inteligencia psicomotriz: importancia y relación. CIENCIA UNEMI, 8(E-15), 110-118. https://doi.org/10.29076/issn.25287737vol8iss15.2015pp110-118p

Battro, A. M. (2000). Half a brain is enough. The story of Nico. Cambridge University Press.

Bonilla, D., Guerrero, A., y López, O. E. (2018). Métodos para medir la calidad del servicio turístico en empresas de alojamiento. Revista Ciencia \& Tecnología, 18(19), 165-180. https:// doi.org/10.47189/rcet.v18i19.186

Bonilla, D. M., Pérez, M. B., Jami, P., y Camacho, M. A. (2019). Proyectos de estimulación temprana: Características esenciales desde la visión meta analítica. EduSol, 19(67), 27-40. http://edusol.cug.co.cu

Bowers, J. S. (2016). The practical and principled problems with educational neuroscience. Psychological Review, 123(5), 600-612. https://doi. org/10.1037/rev0000025

Bozkurt, G. (2017). Social constructivism: Does it succeed in reconciling individual cognition with social teaching and learning practices in mathematics? Journal of Education and Practice, 8(3), 210-218.

Bravo, L. (2015). Psicología cognitiva y neurociencias de la educación en el aprendizaje del lenguaje escrito y de las matemáticas. Revista de Investigación en Psicología, 17(2), 25-37. https://doi.org/10.15381/rinvp. $\underline{\mathrm{v} 17 \mathrm{i} 2.11256}$

Bruer, J. T. (2016). Neuroeducación: Un panorama desde el puente. Propuesta Educativa, 25(46), 14-25.

Bruner, J. S. (1995). Desarrollo cognitivo y educación. Ediciones Moratta, S. L.

Bueno, D. (2017). Neurociencia para educadores. Octaedro.

Bueno, D. (2018). Epigenoma, para cuidar tu cuerpo y tu vida. Plataforma Editorial.

Chavarría, M. (1982). La estimulación temprana: Apuntes sobre sus fundamentos teóricos, bases empíricas y raíces socio-históricas: elementos para una discusión. Revista de Ciencias Sociales, 23, 41-56.

Coelho, F. A. (2017). Neurociencia: Un encuentro posible con la psicología humanística. Revista de Ciencias Sociales (Ve), XXIII(1), 58-66.

Contreras, D. (2020). La estimulación temprana: Una propuesta desde la motricidad como dimensión humana [Tesis de pregrado, Universidad Pedagógica Nacional]. http:// repository.pedagogica.edu.co/ handle/20.500.12209/12340

Dastpak, M., Behjat, F., y Taghinezhad, A. (2017). Un estudio comparativo de las perspectivas de Vygotsky sobre el desarrollo del lenguaje infantil con el nativismo y el conductismo. International Journal of Languages' Education and Teaching, 5(2), 230238. https://files.eric.ed.gov/fulltext/ ED574953.pdf

Diamond, A., y Ling, D. (2016). Conclusions about interventions, programs, and approaches for improving executive functions that appear justified and those that, despite much hype, do not. Developmental Cognitive Neuroscience, 18(1), 34-48. https:// doi.org/10.1016/j.dcn.2015.11.005 
Flórez, J. (2015). Discapacidad intelectual y neurociencia. Revista Síndrome de Down, 32(124), 2-14.

Friedrich, G., y Preiss, G. (2003). Neurodidáctica. Mente y Cerebro, (4), $39-45$.

Guerra, M. E., Zuluaga, A. D., y Saravia, L. A. (2019). Música vallenata, instrumento pedagógico en el proceso de aprendizaje universitario. Revista de Ciencias Sociales (Ve), XXV(1), 59-70.

Guillen, L., Rojas, L., Formoso, A. A., Contreras, L. M., y Estevez, M. A. (2018). Influencia de la estimulación temprana en el desarrollo sensoriomotriz de niños de cuatro a seis años: Una visión desde el Karate Do. Retos, 35, 147-155. https://doi.org/10.47197/ retos.v0i35.63104

Hernández, R., Méndez, S., Mendoza, C. P., y Cuevas, A. (2017). Fundamentos de investigación. McGraw-Hill/ Interamericana de España.

Hernández-Sampieri, R., y Mendoza, C. (2018). Metodología de la investigación: Las rutas cuantitativa, cualitativa y mixta. McGraw-Hill/ Interamericana Editores.

Illes, J. (2005). Neuroethics: Defining the issues in theory, practice and policy. Oxford University Press. https://doi.org/10.1093/acprof:o so/9780198567219.001.0001

Jadán-Guerrero, J., Guevara, C., Lara-Alvarez, P., Sánchez-Gordon, S., CalleJimenez, T., Salvador-Ullauri, L., Acosta-Vargas, P., y Bonilla-Jurado, D. (2020). Building hybrid interfaces to increase interaction with young children and children with special needs. In I. Nunes (Ed.), Advances in Human factors and systems interaction. AHFE 2019. Advances in intelligent systems and Computing, Vol 959 (pp. 306-314). Springer, Cham. https://doi.org/10.1007/978-3-030$\underline{20040-4 \quad 28}$

Jami, L. P., Caisapanta, N. E., Zambrano, R. N., y Bonilla, D. M. (2020). La matrogimnasia y el desarrollo motor en niños entre 7 y 8 años con parálisis cerebral. Retos, 41, 171-181. https:// doi.org/10.47197/retos.v0i41.82765

Labov, W. (1969). The logic of nonstandard English. Monograph series on Languages and Linguistics. Georgetown University School of Languages and Linguistics.

Martínez. A., Quintero, G., y Ruíz, Y. (2013). La importancia del lenguaje en los procesos de aprendizaje. Vanguardia Psicológica Clínica Teórica y Práctica, 4(1), 17-30.

Martínez, S. Y. (2020). La estimulación temprana del área socio emocional para el desarrollo afectivo en los niños de 0-5 años de edad [Tesis de maestría, Universidad Laica Vicente Rocafuerte]. http://repositorio.ulvr. edu.ec/handle/44000/3663

Megías, A. M., y Lozano, L. (2019). Desarrollo socioafectivo. Editorial Editex.

Mensah, E. (2015). Exploring constructivist perspectives in the college classroom. SAGE Open, 5(3), 1-14. https://doi. org/10.1177/2158244015596208

Mora, F. (2017). Neuroeducación: Solo se puede aprender aquello que se ama. Alianza Editorial.

Nizama, M. (2015). Niveles de conocimiento sobre neurociencia y su aplicación en los procesos educativos. In Crescendo, 6(2), 104-113. https://doi. org/10.21895/incres.2015.v6n2.10

Ortiz, A. (2005). Modelos pedagógicos: Hacia una escuela del desarrollo integral. Editorial CEPEDID.

Piaget, J. (1984). El lenguaje y el pensamiento del niño pequeño. Ediciones Paidós. 
Pinos, G., Ayala, D., y Bonilla, D. (2018). Desarrollo del pensamiento lógicomatemático a través de juegos populares y tradicionales en niños de educación inicial. Revista Científica Ciencia y Tecnología, 18(19). https:// doi.org/10.47189/rcct.v18i19.190

Rodríguez, G. E., Orozco, S. P., y Rodríguez, Y. (2016). Deficiencias del lenguaje infantil. Tipo trastorno del lenguaje. Revista ÁRETE, 16(2), 31-41.

Salamanca, L., y Sánchez, M. V. (2018). Desarrollo cognitivo y motor. Editorial Editex.

Salas, R. (2003). Does education really need neuroscience? Estudios pedagógicos, (29), 155-171. http://dx.doi.org/10.4067/S0718$\underline{07052003000100011}$

Stewart, W. (1969). On the use of negro dialect in the teaching of reading. In J. C. Baratz y R. W. Shuy (Eds.), Teaching Black children to read (pp. 156-219). Center for Applied Linguistics.

Stodolsky, S., y Lesser, G. (1967). Learning patterns in the disadvantaged. Harvard Educational Review, 37(4), 546-593. https://doi.org/10.17763/ haer.37.4.p7n62537u638j165

Terranova, A. E., Viteri, E. A., Medina, G. M., y Zoller, M. J. (2019). Desarrollo cognitivo y funcionalidad familiar de infantes en las comunidades urbanomarginales de Ecuador. Revista de Ciencias Sociales (Ve), XXV(E-1), 330-340. https://doi.org/10.31876/rcs. $\underline{\mathrm{v} 25 \mathrm{i} 1.29626}$

Uscategui, M. Y., y Boscan, A. (2017). Neuroeducación: Un enfoque motivador para el proceso de aprendizaje del cálculo inicial. Eco Matemático, 8(S1), 7-9. https://doi. org/10.22463/17948231.1375

Velásquez, Y. E. (2016). Estimulación temprana y desarrollo cognitivo [Tesis de pregrado, Universidad Rafael Landívar]. http://recursosbiblio. url.edu.gt/tesisjcem/2016/05/22/ Velasquez-Yeritza.pdf

Véliz, A. L. (2019). La estimulación sensorial y su incidencia en el desarrollo de las habilidades cognitivas en niños de 3 años en la Unidad Educativa "San Jacinto", Guayaquil, periodo lectivo 2019-2020 [Tesis de pregrado, Universidad Laica Vicente Rocafuerte]. http://repositorio.ulvr. edu.ec/bitstream/44000/3426/1/TULVR-3006.pdf

Vygotsky, L. S. (1995) Pensamiento y lenguaje: Teoría del desarrollo cultural de las funciones psiquicas. Ediciones Fausto. 